\title{
TREATMENT OF CORNEAL INFECTION WITH PS. PYOCYANEA BY SUBCONJUNCTIVAL INJECTION OF POLYMYXIN E*
}

\author{
BY \\ D. IAINSLIE \\ Institute of Ophthalmology, London
}

THE properties of polymyxin in relation to ocular therapeutics have recentl ${ }^{\bar{\omega}}$ been discussed at some length; the penetration of this antibiotic into the eye of the rabbit after subconjunctival injection was estimated and the results obtained in the treatment of corneal infection experimentally produced with Ps. pyocyanea have been reported (Ainslie and Smith, 1951).

It is well known that this type of ocular infection runs an unusually serious course in man. In view of its resistance to the methods of treatment -including the usual antibiotics-hitherto employed clinically, in contras to the satisfactory results obtained during investigations on animals, it was decided to proceed with clinical trials. The treatment with Polymyxin E of six proved cases of corneal infection due to Ps. pyocyanea and of two furtheg cases showing the clinical features of such infection but without cultura楚 confirmation are reported below.

\section{Preparation}

The Polymyxin E† was prepared for injection by dissolving the contents of one vial of $1,000,000$ units in $1 \mathrm{ml}$. of an aqueous solvent consisting of mydricaine $0.5 \mathrm{ml}$. and sterile distilled water $0.5 \mathrm{ml}$. In order to obtain a perfect solution it was necessary to allow a용 long as half an hour for the polymyxin to dissolve. This solution was found to be stablefor at least a week. The dosage used was $0.20-0.25 \mathrm{ml}$. (200,000 to 250,000 units).

\section{Tolerance}

No permanent ill effects from the injections were noted. Chemosis soon subs sided when treatment was stopped and no conjunctival scarring could be detected: One patient (Case 3 ) received a total of 21 injections, but despite this the conjunctiv settled entirely and now appears normal.

Considerable pain was experienced by most patients after the injections. Fou心 complained of extremely severe pain and had to be given strong analgesics (either pethidine or omnopon) with each dose. Two said that the pain was moderate an\& one that there was no pain at all. The remaining patient (Case 6) had had aep alcohol injection into his trigeminal ganglion and was therefore insensitive. Ip general it appears that the injections cause pain for about 2 hours after administra tion.

* Received for publication February 2, 1953.

+ Supplied by the Wellcome Research Institute in vials of $1,000,000$ units. 


\section{Treatment of Cases}

In all the cases described here the pupils were kept fully dilated with atropine throughout the treatment, and the eyes were padded during the period of the injections.

\section{Case 1. A man aged 40.}

History and Previous Treatments.-On January 25, 1952, his right cornea was scratched by a baby's finger nail. It appeared to heal but 2 weeks later the cornea broke down and a severe hypopyon ulcer formed. A culture was taken and 500,000 units of penicillin given subconjunctivally. The following day there was no improvement, and, as the culture showed Ps. pyocyanea, a subconjunctival injection of $0.5 \mathrm{~g}$. streptomycin was given, followed by a further dose the next day. There was marked improvement and the streptomycin was discontinued. Chloramphenicol ointment was applied to the conjunctival sac four times daily. The infection seemed to be overcome, but, as there was extensive corneal vascularization, cortisone $10 \mathrm{mg}$. was injected subconjunctivally on March 3, 1952, and this was followed by cortisone drops. On March 15 the eye again became very painful.

Examination.-On March 18, 1952, there was an ulcer involving almost the whole of the cornea with extensive infiltration. A hypopyon filled more than half the anterior chamber. There was gross chemosis and $2 \mathrm{~mm}$. proptosis.

Therapy.-After a conjunctival swab had been taken, 200,000 units Polymyxin E were injected subconjunctivally. The following day the culture from the swab showed growth of Ps. pyocyanea and Staph. aureus. A further injection of 200,000 units polymyxin was given and aureomycin ointment was applied to the conjunctival sac three times daily. This treatment was continued, polymyxin injections being given daily, until March 31 .

Result.-Until March 27 there was a steady improvement, and on this date the hypopyon was less than $2 \mathrm{~mm}$. Despite the continuation of treatment the condition gradually worsened over the following 4 days, and, as the proptosis had increased, the eyeball was eviscerated on April 1, 1952.

This was the first patient to be treated. The infection could not be more than temporarily controlled, but, in the face of so extensive an infection, even a temporary improvement was considered encouraging.

\section{Case 2. A man aged 23.}

History and Previous Treatment.-The patient had had occasional foreign bodies on the cornea between 1946 and 1951. On April 21, 1952, he complained that for 2 days the right eye had been feeling as though something was in it and had become very red. A central corneal ulcer 6-7 mm. in diameter was noted with oedema of the whole cornea. There was a hypopyon of about $4 \mathrm{~mm}$. A culture was taken on the evening of April 23 ; systemic chloramphenicol was started, and 250,000 units penicillin was given subconjunctivally. A further 200,000 units penicillin was given the next day and the systemic chloramphenicol was continued. On April 25 the culture showed a growth of Ps. pyocyanea.

Examination.-On April 26 there was a corneal ulcer 5-6 mm. in diameter and infiltration extending $2 \mathrm{~mm}$. round this. The anterior chamber was half filled with pus.

Therapy.-250,000 units polymyxin was given subconjunctivally and the dose was repeated on April 27 and 28. The next day there was still marked corneal infiltration but the hypopyon had disappeared. Polymyxin injections were repeated on April 30 and May 1. No further polymyxin was given but aureomycin ointment was applied twice daily. 
Result.-The patient was discharged on May 21. There was no ulceration but moderate corneal infiltration.

On December 11, 1952, the eye was white and quiet. There was a nebula, mainly of the lower half of the cornea, and moderate superficial vascularization. Visual acuit 9 in the right eye was $6 / 24$.

\section{Case 3. A boy aged 16.}

History and Previous Treatments.-On April 28, 1952, a foreign body blew into th right eye. The foreign body was removed the same day but the eye became very sor $\bar{\emptyset}$ and red. On April 30 a large corneal ulcer was present. The patient was given 500,006 units penicillin subconjunctivally and this was repeated the following day. In addition. 0.5 g. chloramphenicol was given 6-hourly. On May 1 culture revealed a heavy growth of Ps. pyocyanea.

Examination.-On May 2 there was an area of infiltration involving three-quarters the cornea and a central ulcer $5 \mathrm{~mm}$. in diameter. There was much purulent discharge The anterior chamber was half full of pus.

Therapy.-Polymyxin 250,000 units was given by subconjunctival injection and the systemic chloramphenicol was continued. On May 3 the discharge was less and the्g hypopyon smaller, but the corneal appearance had not altered. A further injection of 250,000 units polymyxin was given. By the next day there was no discharge and the hypopyon was reduced to $1 \mathrm{~mm}$. The ulcer was smaller. In addition to the injection polymyxin ointment 1 per cent. was now applied twice daily to the conjunctival sac. Daily subconjunctival injections and twice-daily applications of polymyxin ointment were్ continued until May 8.

All treatment with polymyxin was then stopped as the lesion appeared to be healed Aureomycin ointment was applied three times daily to keep the conjunctival sac clear of secondary infection. The eye continued to settle until May 16, when corneal infiltratiod increased and a small central stain appeared. A hypopyon of $3 \mathrm{~mm}$. formed rapidly@ Aureomycin ointment was continued and another subconjunctival injection of polymyxint 250,030 units was given at once. By the following day the hypopyon was reduced t $\sigma^{\circ}$ $1 \mathrm{~mm}$. Daily injections of polymyxin 250,000 units were continued until May 27, an then reduced to every second day, the last injection being given on May 31 .

Result.-The patient was discharged from hospital on June 4 with a dense centra nebula and superficial vascularization of the cornea. On August 6 the eye was comforto able and entirely quiet. There was a dense firm nebula with slight vascularization: Visual acuity was counting fingers at $2 \mathrm{ft}$.

\section{Case 4. A woman aged 48.}

History and Previous Treatments. - Since the age of 5 the patient had had poor visio? due to corneal opacities. On March 27 a left penetrating keratoplasty was performed. The् stitches were removed on April 3 and the patient was discharged from hospital on April $17_{\mathrm{N}}^{\circ}$ On April 24 she was readmitted, having a hypopyon ulcer. Culture taken from the्ष cornea showed Ps. pyocyanea, sensitive to streptomycin. Polymyxin sensitivity was nof tested. Subconjunctival streptomycin was given daily and aureomycin ointment was applied to the conjunctivaı sac. The hypopyon lessened in size but it did not disappeaE and the ulcer remained unchanged. On May 5 the ulcer had again increased in size and the hypopyon was also larger. Reculture showed Ps. pyocyanea to be still presento

Examination.-On May 7 there was a central corneal ulcer 5-6 mm. in diameter, witlo surrounding infiltration, and a $3 \mathrm{~mm}$. hypopyon.

Therapy.-A subconjunctival injection of 250,000 units polymyxin was given. The anterior chamber was opened, the hypopyon evacuated, and the anterior chamber irrigated with polymyxin solution, 200,000 units in $5 \mathrm{cc}$. saline. Daily subconjunctival injection? 
of polymyxin 250,000 units were given until May 18. On May 20 the eye appeared free from infection but culture produced a growth of Staph. aureus. The patient was discharged from hospital and instructed to use aureomycin ointment three times a day.

Result.-On September 9 the eye was quiet but the graft was completely opaque. Visual acuity was perception of light with good projection.

\section{Case 5. A man aged 53.}

History and Previous Treatment.-A parotid tumour had been excised in 1926. It recurred, and several further operations had to be performed. A facial palsy with partial exposure of the cornea occurred. In addition, the cornea was anaesthetic following alcohol injection of the trigeminal ganglion some time previously, for the relief of pain associated with the neoplasm. A corneal ulcer was first noted on July 3. This did not respond to treatment with atropine, albucid, and padding of the eye, but became slowly worse. A small hypopyon formed and a lateral half tarsorrhaphy was performed on August 6.

Examination.-On August 11 there was a corneal ulcer $3 \mathrm{~mm}$. in diameter and deep corneal infiltration about $6 \mathrm{~mm}$. in diameter. The ulcer had the clinical appearance of one due to a Gram-negative bacillus and culture revealed Ps. pyocyanea.

Therapy.-On August 12 250,000 units polymyxin was given subconjunctivally, and thrice daily applications of polymyxin ointment 1 per cent., which had already been started, were continued. Subconjunctival injections of 250,000 units of polymyxin were given on August 14 and 15. The condition was now much improved but there was still some coagulated exudate in the anterior chamber and the cornea was still stained. Further culture from the ulcer revealed Staph. aureus sensitive to chloramphenicol, but no Ps. pyocvanea was grown.

A further injection of polymyxin 250,000 units was given on August 16, and chloramphenicol ointment was applied to the conjunctival sac. This ointment was continued, and subconjunctival injections were repeated every second day until August 26. There was now no hypopyon but still moderate corneal infiltration.

The polymyxin was stopped but the chloramphenicol ointment was continued. On September 5 a hyphaema developed, but the eye did not appear to be infected. On September 13, however, the hyphaema appeared to be mixed with pus, and polymyxin injections were given on September 13 and 15 as it was thought that a recurrence of the infection might be imminent. The eye then gradually quietened but the hyphaema did not absorb. On October 25 an anterior chamber washout was performed.

Result.- Since this date there has been no sign of recurrence of the infection but there is still a hyphaema present and the cornea is rough with a variable amount of staining. Visual acuity is perception of light.

It would seem that this chronic residual ulceration is of the neuroparalytic type associated with the absence of trigeminal sensation. It is certain, from the chronic nature of the present ulceration, that there is no further pyocyaneus infection. Unfortunately, because of the progress of the parotid carcinoma, the patient's general condition is very poor and only simple palliative treatment is being applied to the eye.

\section{Case 6. A man aged 32.}

History and Previous Treatment.-On September 9 the patient's eye became painful and red and he attended hospital on September 20, 1952, when a large corneal infiltrate with corneal abscess $5 \mathrm{~mm}$. in diameter was present in the lower half of the cornea. There were cells in the anterior chamber but no hypopyon. A culture was taken, 500,000 units penicillin were given subconjunctivally and penicillin ointment applied. On the following 
day the infiltrate had increased and there was a small hypopyon. The eye was by now very painful. The culture gave a good growth of Ps. pyocyanea. Streptomycin $0.5 \mathrm{~g}$. was given subconjunctivally and chloramphenicol 0.5 g. 6-hourly. On September 22 the in vitro sensitivity of the organism to antibiotics was known. It was insensitive too penicillin, aureomycin, and chloramphenicol, and moderately sensitive to polymyxin, streptomycin, and terramycin. Accordingly, terramycin ointment was applied to the conjunctival sac 3-hourly, and a further subconjunctival injection of $0.5 \mathrm{~g}$. streptomycin? was given. Streptomycin injections were repeated on September 23, 24, 25, and 27, but: the condition became gradually worse.

Examination.-On September 29 the whole lower half of the cornea was infiltrated ando there was $2-3 \mathrm{~mm}$. of hypopyon. The eye was grossly injected and there was chemosis? and oedema of the lids.

Therapy.-The terramycin ointment was continued and a subconjunctival injection of 200,000 units polymyxin was given, and repeated the following day. On September 3, so0 great an improvement was noted that injections were discontinued, but on September $5_{\omega}^{\circ}$ the condition seemed a little worse, and 200,000 units polymyxin was given subconjuncti- -0 vally. The dose was repeated on September 8 and 13.

Result.-The eye settled well and the patient was discharged on September 18. There was no hypopyon, but a vascularized corneal nebula was present below and there was slight corneal oedema above. On November 20 the eye was quiet, with a dense corneal $\vec{c}$ nebula below, and there was no sign of recurrence of infection. Visual acuity was hand $\frac{\bar{\sigma}}{\bar{\sigma}}$ movements.

The remaining cases showed the characteristic diffuse corneal infiltration found in infections due to Gram-negative bacilli (Bignell, 1951 ; Sorsby and others, 1952) but cultural confirmation was lacking.

\section{Case 7. A man aged 70.}

History and Previous Treatment.-The patient had chronic glaucoma and a left anterior sclerotomy had been performed in 1950 . The eye did very well until on June 20, 1952, it became red and sore and on the following day it was found that the conjunctiva over the scleral aperture was infected and there was a hypopyon of $3 \mathrm{~mm}$. A subconjunctivalo injection of penicillin 200,000 units was given at once and systemic treatment witho chloramphenicol $750 \mathrm{mg}$. 6-hourly was started.

The treatment, including daily subconjunctival injections of penicillin, was continued $\overline{3}$. until June 24, when as there had been no improvement an anterior chamber washout wasi performed. The following day the hypopyon had re-formed and treatment was started $₹$ with aureomycin ointment 1 per cent. However, the eye became steadily worse. Severalo cultures from the conjunctival sac had been taken but only Staph. albus was grown.

Examination.-On June 27 the upper bulbar conjunctiva was oedematous and appeared infected and there was infiltration of the upper part of the cornea. There was a hypopyon $3.5 \mathrm{~mm}$. in diameter.

Therapy. -250,000 units Polymyxin E were injected subconjunctivally, and the next day the hypopyon had decreased to $1 \mathrm{~mm}$. A further dose of 250,000 units polymyxin was injected and by June 29 the hypopyon had disappeared. On June 30 and July 1 the polymyxin injections were repeated. No further polymyxin was given, but aureomycin ${ }^{2}$ ointment, which had been applied throughout, was continued until July 14.

Result.-The patient was discharged from hospital on July 16, there being no sign of infection. A $1 \mathrm{~mm}$. hyphaema, lens opacities, and posterior synechiae were present $\stackrel{\vec{\Omega}}{\mathrm{Q}}$ The eye gradually settled down and is now quiet with normal tension, though the visuab acuity is only hand movements. 


\section{Case 8. A man aged 39.}

Case History and Previous Treatment.-A corneal foreign body was removed on August 7, 1952. The eye became very red and sore and on August 9 there was a large corneal infiltrate and a $1 \mathrm{~mm}$. hypopyon. A culture was taken, and only Staph. albus was grown, though the clinical appearance was that of a Ps. pyocyanea infection. Streptomycin $0.5 \mathrm{~g}$. was given subconjunctivally and repeated on August 10 and 11 . Streptomycin drops 10,000 units per $\mathrm{ml}$. were instilled half-hourly during this time, but by August 12 the hypopyon had increased to $2.5 \mathrm{~mm}$. Further subconjunctival injections and streptomycin drops were given but the eye became worse.

Examination.-On August 15 an intracorneal abscess $5 \mathrm{~mm}$. in diameter was present, but there was no actual ulceration. The anterior chamber was half full of pus. The streptomycin drops were continued and 200,000 units polymyxin given subconjunctivally in addition. On August 16 the hypopyon was much reduced; the polymyxin injection was repeated, and again on the following day, when there was still corneal infiltration but no hypopyon.

Result.-On August 23 the eye was very injected but the cornea was bright and clearing, and the patient was discharged from hospital.

Relapse.-The eye was slightly irritable but remained about the same until September 18, when it again became very painful. The next day a hypopyon of $2 \mathrm{~mm}$. diameter was again apparent and there was central active corneal infiltration. 200,000 units of polymyxin was again given subconjunctivally and the dose was repeated daily until September 23.

Result.-The hypopyon disappeared and the infiltration lessened, leaving a dense central nebula. The patient was discharged from hospital on September 28 with no hypopyon but a faint flare. On November 6 the eye was almost white, and there was no flare but a dense nebula. Visual acuity was counting fingers.

\section{Discussion}

In five of the six cases of proved pyocyaneus ulceration which were treated, the infection was overcome by subconjunctival injections of polymyxin. When treatment was started, every case appeared to be progressing rapidly towards loss of the eye.

The only failure was an exceptionally advanced case in which the whole cornea was already involved when first seen, and even in this instance there was initial improvement. The failure to maintain the improvement could be accounted for by the fact that many subconjunctival injections of penicillin and streptomycin had already been given and the absorption of the polymyxin was probably unsatisfactory owing to the scarred condition of the subconjunctival tissues. Before any polymyxin was given the conjunctiva was already oedematous and friable.

Both the patients in whom the corneal infection was diagnosed as due to Ps. pyocyanea on clinical grounds alone were cured.

In two of the seven successfully treated cases, the infection recurred after cessation of the first course of treatment, but was rapidly brought under control again by a second course. This emphasizes the difficulty with which infection of the cornea due to Ps. pyocyanea is eradicated entirely, and suggests that treatment should always be continued for several days after the clinical appearance indicates that the infection has been overcome. 
Much tissue necrosis is always associated with corneal infections witl pyocyaneus, and sometimes a definite intracorneal abscess develops. It wa䒠 found during the experimental series that, as the pyocyaneus infection was overcome, there was a tendency for the still necrotic cornea to be invaded. by secondary infective organisms, particularly the staphylococci.

It should be borne in mind that, despite its powerful activity against the Gram-negative bacilli, polymyxin is completely ineffective against the coccis and great care must therefore be taken to keep the conjunctival sac as free as possible from these organisms during treatment. A suitable ointments such as aureomycin, chloramphenicol, or terramycin, should be applied frequently, and all discharge should be removed by irrigation when necessaryes

Aureomycin, chloramphenicol, or terramycin ointment was applies. liberally in seven of the eight cases reported, but no apparent improvementw followed these applications alone, nor did any improvement follow systemie chloramphenicol therapy alone.

The danger of secondary infection is of course not so great when the Gram-negative infection is treated with streptomycin, since this antibiotic is also effective against most coccal organisms. Gram-negative organisms however, are usually found to be more sensitive to polymyxin than to streptomycin (Brownlee and Bushby, 1948). Also, organisms resistant t $\Theta$ polymyxin are produced only with difficulty, whereas organisms very readil become insensitive to streptomycin (Finland and others, 1946 ; Paine an Finland, 1948).

In four of the cases described above, streptomycin had been applied bo subconjunctival injection before polymyxin was tried. The streptomycio therapy was followed by temporary improvement in two cases (Cases 1 and 4) but in the other two (Cases 6 and 8) it produced no change. Immediate improvement followed the polymyxin injections in all cases.

In this series of cases, the visual acuity remaining at the end of treatmen was poor, in no case better than 6/24 and in most considerably less. How ever, with the exception of Case 5 (neuroparalytic keratitis) and Case (infected sclerotomy), the intra-ocular structures appear to be healthy, and the poor visual acuity entirely due to the density of the corneal nebulae.

\section{Summary}

Eight cases of corneal ulceration treated with polymyxin $\mathrm{E}$ are describe and the results discussed.

I am greatly indebted to Dr. Charles Smith for the bacteriological examinations. I should like to thank the surgeons of the Moorfields, Westminster and Central Eye Hospital for the facilities and assistance they have afforded me.

\section{REFERENCES}

AINSLIE, D., and SmIth, C. H. (1952). British Journal of Ophthalmology, 36, 352.

BigNell, J. L. (1951). Ibid., 35, 419.

BrownleE, G., and Bushby, S. R. M. (1948). Lancet, 1, 127.

Finland, M., Murray, R., Harris, H. W., Kilham, L., and Meads, M. (1946). J. Amer. meq Ass., 132, 16.

Paine, T. F., and Finland, M. (1948). J. Bact., 56, 207.

SORSBY, A., UNGAR, J., and BAILEY, N. L. (1952). Brit. med. J., 1, 119. 